\title{
A note on the Klein-Gordon equation in the background of a rotating black hole
}

\author{
Horst R. Beyer ${ }^{\mathrm{a})}$ \\ Center for Computation and Technology (CCT), Louisiana State University (LSU), \\ 328 Johnston Hall, Louisiana 70803, USA and Max Planck Institute for Gravitational \\ Physics, Albert Einstein Institute (AEI), Am Mühlenberg 1, D-14476 Potsdam, Germany
}

(Received 26 June 2008; accepted 4 November 2008; published online 6 January 2009)

This short paper should serve as a basis for further analysis of a previously found new symmetry of the solutions of the wave equation in the gravitational field of a Kerr black hole. Its main new result is the proof of essential self-adjointness of the spatial part of a reduced normalized wave operator of the Kerr metric in a weighted $L^{2}$-space. As a consequence, it leads to a purely operator theoretic proof of the well posedness of the initial value problem of the reduced Klein-Gordon equation in that field in that $L^{2}$-space and in this way generalizes a corresponding result of Kay ["The double-wedge algebra for quantum fields on Schwarzschild and Minkowski spacetimes," Commun. Math. Phys. 100, 57 (1985)] in the case of the Schwarzschild black hole. It is believed that the employed methods are applicable to other separable wave equations. () 2009 American Institute of Physics.

[DOI: $10.1063 / 1.3037327]$

\section{GENERAL INTRODUCTION}

The linearized stability of the Schwarzschild black hole follows by combining the ReggeWheeler-Zerilli-Moncrief decomposition of gravitational perturbations of the Schwarzschild metric $^{16,19,13}$ with a result by Kay and Wald ${ }^{12}$ that proves the boundedness of all solutions of the wave equation corresponding to $C^{\infty}$-data of compact support. The proof of the last rests on the positivity of the conserved energy.

The question of the linearized stability of the Kerr black hole is still an open problem whose outcome is of major importance to general relativity. In comparison to the case of the Schwarzschild black hole, the solution to this problem is considerably more complicated. Mainly, this is due to two facts. First, a decomposition comparable to that of Regge-Wheeler-Zerilli-Moncrief does not yet exist in this case, although the recent finding in Ref. 6 gives hope that such a decomposition might exist. In contrast, a partial decomposition based on the Newman-Penrose formalism depends on the choice of a tetrad field, i.e., is gauge dependent even under "small" coordinate transformations. ${ }^{2}$ Second, a conserved energy for the solutions of the wave equation exists, but the energy density is negative inside the ergosphere. This fact excludes at least a direct application of the so-called "energy methods" to a proof of stability of the solutions. The total energy could be finite while the field still might grow exponentially in parts of the space-time. But recently a local stability result has been proved that the restrictions of the solutions of the wave equation to compact subsets $K$ in space are elements of $L_{C}^{\infty}(K)$ with a norm converging to zero for $t \rightarrow \infty$. Because of the absence of a decomposition of the Regge-Wheeler-Zerilli-Moncrief type, the question of applicability of the last and other similar results to the question of linearized stability of the Kerr metric is still open.

As mentioned above, Ref. 6 contains the surprising find of a new symmetry operator that

${ }^{a)}$ Electronic mail: horst@cct.lsu.edu. 
commutes with a normalized ${ }^{1}$ form of the wave operator in a Kerr background. Contrary to previously known symmetry operators for the wave operator, this operator contains only a partial time derivative of the first order, but not of higher order. As a consequence, in formulations of the initial value problem for the wave equation in terms of first order systems of partial differential equation and related formulations such as Ref. 4 , this operator leads to an operator $\hat{S}$ that formally ${ }^{2}$ commutes with the infinitesimal generator $G$ of time evolution and, therefore, is a candidate for the generator of a strongly continuous semigroup or group of symmetries. In precise terms, $\hat{S}$ should lead to an operator $\mathcal{S}$ that intertwines with the operators from the strongly continuous one-parameter group $T:[0, \infty) \rightarrow L(Y, Y)$ generated by $G,{ }^{5,10}$ i.e., which is such that

$$
\mathcal{S} T(t) \supset T(t) \mathcal{S}
$$

for all $t \geq 0$. Here $Y$ denotes the space of data for the wave equation which is a complex Hilbert space. ${ }^{4}$ The goal of the present note is to lay part of the foundation for a proof of the last in providing the new proof of the essential self-adjointness of the spatial part of the reduced normalized wave operator $A_{0}$ of the Kerr metric in a weighted $L^{2}$-space. In addition, it is believed that the employed method in this might be applicable to other separable wave equations. In this connection, it should be remarked that the operator $A_{0}$ is essentially different from the operator $V$ considered in Ref. 1. The differences are easily seen as a consequence of the different approaches of both papers to the formulation of a well-posed initial value formulation for the Klein-Gordon field on a Kerr background. In addition, unlike the case of $A_{0}$, the connection of the essential self-adjointness of $V$ to a well-posed initial value formulation for the Klein-Gordon equation is still open. Finally, there are differences in the approaches of both papers in their proofs of essential self-adjointness. Also, the method of proof in this paper is new.

As a consequence, we also arrive at a purely operator theoretic proof of the well posedness of the initial value problem of the reduced normalized Klein-Gordon equation in the gravitational field of a Kerr black hole in the weighted $L^{2}$-space. The last space was already used in Ref. 4. Also, the last reference gives such a proof, but under the assumption that physical boundary conditions lead to the Friedrichs extension of the operator that is obtained from the spatial part of the reduced normalized wave operator of the Kerr metric by restriction to $C^{\infty}$-functions with compact support. In particular, the results in this note prove that this assumption is justified.

\section{THE MATHEMATICAL SETTING}

In Boyer-Lindquist coordinates, ${ }^{3}(t, r, \theta, \varphi): \Omega \rightarrow \mathbb{R}^{4}$, the Kerr metric $g$ is given by

$$
\begin{aligned}
g= & \left(1-\frac{2 M r}{\Sigma}\right) d t \otimes d t+\frac{2 M a r \sin ^{2} \theta}{\Sigma}(d t \otimes d \varphi+d \varphi \otimes d t)-\frac{\Sigma}{\Delta} d r \otimes d r-\Sigma d \theta \otimes d \theta \\
& -\frac{\Delta \bar{\Sigma}}{\Sigma} \sin ^{2} \theta d \varphi \otimes d \varphi
\end{aligned}
$$

where $M$ is the mass, $a \in[0, M]$ is the rotational parameter, and

\footnotetext{
${ }^{1}$ In the following, the term "normalized" means multiplication from the left by the factor $1 / g^{00}$ in Boyer-Lindquist coordinates. For instance, such a "normalization" takes place if the Klein-Gordon equation is solved for the second order time derivative of the unknown. Commonly, the last is a first step in the formulation of an initial value problem for the solutions of Klein-Gordon equations in curved space-times.

${ }^{2}$ That is, on the level of tuples of functions that are differentiable to sufficiently high order.

${ }^{3}$ See Ref. 7. If not otherwise indicated, the symbols $t, r, \theta, \varphi$ denote coordinate projections whose domains will be obvious from the context. In addition, we assume the composition of maps, which includes addition, multiplication, and so forth, always to be maximally defined. For instance, the sum of two complex-valued maps is defined on the intersection of their domains. Finally, we use Planck units where the reduced Planck constant $\hbar$, the speed of light in vacuum $c$, and the gravitational constant $\gamma$, all have the numerical value of 1 .
} 


$$
\begin{gathered}
\Delta:=r^{2}-2 M r+a^{2}, \quad \Sigma:=r^{2}+a^{2} \cos ^{2} \theta, \\
\bar{\Sigma}:=\frac{\left(r^{2}+a^{2}\right) \Sigma+2 M a^{2} r \sin ^{2} \theta}{\Delta}=\frac{\left(r^{2}+a^{2}\right)^{2}}{\Delta}-a^{2} \sin ^{2} \theta, \\
r_{+}:=M+\sqrt{M^{2}-a^{2}}, \quad \Omega:=\mathbb{R} \times\left(r_{+}, \infty\right) \times(-\pi, \pi) \times(0, \pi) .
\end{gathered}
$$

In these coordinates, the reduced wave equation, governing solutions of the form

$$
\psi(t, r, \theta, \varphi)=\exp (\operatorname{im} \varphi) u(t, r, \theta),
$$

where $m$ runs through all integers, is given by

$$
\frac{\partial^{2} u}{\partial t^{2}}+\frac{1}{\bar{\Sigma}} \cdot\left(i \frac{4 m M a r}{\Delta} \frac{\partial u}{\partial t}-\frac{\partial}{\partial r} \Delta \frac{\partial}{\partial r}-\frac{m^{2} a^{2}}{\Delta}-\frac{1}{\sin \theta} \frac{\partial}{\partial \theta} \sin \theta \frac{\partial}{\partial \theta}+\frac{m^{2}}{\sin ^{2} \theta}\right) u=0 .
$$

The spatial part of the reduced normalized wave operator is given by

$$
D_{r \theta}^{2} f:=\frac{1}{\bar{\Sigma}}\left(-\frac{\partial}{\partial r} \Delta \frac{\partial}{\partial r}-\frac{m^{2} a^{2}}{\Delta}-\frac{1}{\sin \theta} \frac{\partial}{\partial \theta} \sin \theta \frac{\partial}{\partial \theta}+\frac{m^{2}}{\sin ^{2} \theta}\right) f
$$

for every $f \in C^{2}(\Omega, C)$. In particular, $D_{r \theta}^{2}$ is singular since the continuous extensions of the coefficients of its highest (second) order radial derivative vanish on the horizon $\left\{r_{+}\right\} \times[0, \pi]$. We represent the operator $D_{r \theta}^{2}$ as the operator $A$ below in the weighted $L^{2}$-space $X$ defined by

$$
X:=L_{C}^{2}\left(\Omega, g^{00} \sqrt{-|g|}\right) .
$$

Here $|g|$ denotes the determinant of the matrix $g_{a b}$. Note that

$$
g^{00} \sqrt{-|g|}=\bar{\Sigma} \sin \theta
$$

is singular at the horizon. Hence the elements of $X$ vanish there in the mean. In the limit $a \rightarrow 0$, this weight reduces to the one that is commonly used in the stability discussion of the Schwarzschild metric. $^{17,11}$

\section{PROPERTIES OF THE SPATIAL PART OF THE REDUCED NORMALIZED WAVE OPERATOR}

In the following, we prove that the operator $A_{0}$ defined below is linear, symmetric, and essentially self-adjoint. ${ }^{4}$

\section{Definition 1:}

(i) We define the domain of $A_{0}$ to consist of all $f \in C^{2}(\bar{\Omega}, \mathrm{C}) \cap X$ satisfying the conditions (a)-(c).

(a) $D_{r \theta}^{2} f \in X$.

(b) There is $R>0$ such that $f(r, \theta)=0$ for all $r>R$ and $\theta \in I_{\theta}:=(0, \pi)$.

(c)

$$
\lim _{r \rightarrow r_{+}} \frac{\partial f}{\partial \theta}(r, \theta)=0
$$

${ }^{4}$ Note that the following operator $A_{0}$ corresponds to the operator $A_{0}+C$ in Ref. 4. 
for all $\theta \in I_{\theta}$.

(ii) For every $f$ in the domain of $A_{0}$, we define

$$
A_{0} f:=D_{r \theta}^{2} f .
$$

Lemma 2: $A_{0}$ is a densely defined, linear, and symmetric operator in X. In addition, $A_{0}$ is semibounded from below with lower bound

$$
\alpha:=-\frac{m^{2} a^{2}}{4 M^{2} r_{+}^{2}} .
$$

Proof: In the following, $\langle\mid\rangle$ denotes the scalar product on $X$. Obviously, the domain of $A_{0}$ is a subspace of $X$ that contains $C_{0}^{2}(\Omega, \mathrm{C})$. Since the last is a dense subspace of $X$, that domain is a dense subspace of $X$. Further, $A_{0}$ is obviously linear. In particular, it follows for $f, g$ from the domain of $A_{0}$ that

$$
\bar{\Sigma} \sin \theta f^{*} A_{0} g=\frac{\partial}{\partial r}\left\{\Delta \sin \theta\left[\left(\frac{\partial f}{\partial r}\right)^{*} g-f^{*} \frac{\partial g}{\partial r}\right]\right\}+\frac{\partial}{\partial \theta}\left\{\sin \theta\left[\left(\frac{\partial f}{\partial \theta}\right)^{*} g-f^{*} \frac{\partial g}{\partial \theta}\right]\right\}+\bar{\Sigma} \sin \theta\left(A_{0} f\right)^{*} g
$$

and hence by Green's theorem that

$$
\left\langle f \mid A_{0} g\right\rangle=\int_{\Omega} \bar{\Sigma} \sin \theta f^{*} A_{0} g d r d \theta=\int_{\Omega} \bar{\Sigma} \sin \theta\left(A_{0} f\right)^{*} g d r d \theta=\left\langle A_{0} f \mid g\right\rangle .
$$

Hence, $A_{0}$ is symmetric. Further, it follows for $f$ from the domain of $A_{0}$ that

$$
\begin{aligned}
\bar{\Sigma} \sin \theta f^{*}\left(A_{0}-\alpha\right) f= & -\frac{\partial}{\partial r}\left(\Delta \sin \theta f^{*} \frac{\partial f}{\partial r}\right)-\frac{\partial}{\partial \theta}\left(\sin \theta f^{*} \frac{\partial f}{\partial \theta}\right)+\sin \theta\left\{\Delta\left|\frac{\partial f}{\partial r}\right|^{2}+\left|\frac{\partial f}{\partial \theta}\right|^{2}\right. \\
& \left.+\left(-\alpha \bar{\Sigma}-\frac{m^{2} a^{2}}{\Delta}+\frac{m^{2}}{\sin ^{2} \theta}\right)|f|^{2}\right\} \geq-\frac{\partial}{\partial r}\left(\Delta \sin \theta f^{*} \frac{\partial f}{\partial r}\right)-\frac{\partial}{\partial \theta}\left(\sin \theta f^{*} \frac{\partial f}{\partial \theta}\right),
\end{aligned}
$$

where it has been used that

$$
\bar{\Sigma} \geq \frac{4 M^{2} r_{+}^{2}}{\Delta}
$$

Hence it follows by Green's theorem that

$$
\left\langle f \mid\left(A_{0}-\alpha\right) f\right\rangle=\int_{\Omega} \bar{\Sigma} \sin \theta f^{*}\left(A_{0}-\alpha\right) f d r d \theta \geq 0
$$

and, finally, that $A_{0}$ is semibounded from below with lower bound $\alpha$.

Remark 3: We note that the domain of $A_{0}$ contains all products $f \otimes\left(P_{l}^{m} \circ \cos \right)$, where $f$ $\in C_{0}^{2}\left(I_{r}, \mathrm{C}\right)$ and $P_{l}^{m}:(-1,1) \rightarrow \mathbb{R}$ is the generalized Legendre polynomial corresponding to $m \in \mathbb{Z}$ and $l \in\{|m|,|m|+1, \ldots\}$.

Theorem 4: $A_{0}$ is essentially self-adjoint.

Proof: According to a well-known criterion for essential self-adjointness, ${ }^{5}$ it follows that $A_{0}$ is essentially self-adjoint if there is $\lambda<-\alpha$ such that the range of $A_{0}-\lambda$ is dense in $X$. The existence of such $\lambda$ will be shown in the following. For this, we note that the elementary inequalities

${ }^{5}$ For example, see Sec. X.1 in Vol. II of Ref. 15. 


$$
\frac{r^{4}}{\Delta} \leq \bar{\Sigma} \leq \frac{4 M^{2}}{r_{+}^{2}} \frac{r^{4}}{\Delta}
$$

imply that the underlying sets of $X$ and $L_{C}^{2}\left(\Omega, r^{4} \sin \theta / \Delta\right)$ are equal and that the corresponding norms that are induced on that common set are equivalent. These facts are basic for the following.

In a first step, we note that the following holds for arbitrary $\lambda \in \mathbb{C}$ and arbitrary $f$ in the domain of $A_{0}$ :

$$
\left(A_{0}-\lambda\right) f=T_{r^{4} /(\Delta \bar{\Sigma})}\left[\frac{1}{r^{4} / \Delta}\left(-\frac{\partial}{\partial r} \Delta \frac{\partial}{\partial r}-\frac{m^{2} a^{2}}{\Delta}-\frac{1}{\sin \theta} \frac{\partial}{\partial \theta} \sin \theta \frac{\partial}{\partial \theta}+\frac{m^{2}}{\sin ^{2} \theta}\right)-\lambda-\lambda T_{\left(\Delta \bar{\Sigma} / r^{4}\right)-1}\right] f,
$$

where $T_{r^{4} /(\Delta \bar{\Sigma})}, T_{\left(\Delta \bar{\Sigma} / r^{4}\right)-1}$ denote the maximal multiplication operators in $X$ with the functions $r^{4} /(\Delta \bar{\Sigma})$ and $\left(\Delta \bar{\Sigma} / r^{4}\right)-1$, respectively. As a consequence of (3), $T_{r^{4} /(\Delta \bar{\Sigma})}$ is defined on the whole of $X$ as well as bounded and bijective. In addition, as a consequence of

$$
0 \leq \frac{\Delta \bar{\Sigma}}{r^{4}}-1 \leq 5 \frac{a^{2}}{M^{2}},
$$

it follows that $T_{\left(\Delta \bar{\Sigma} / r^{4}\right)-1}$ is a bounded positive self-adjoint operator on $X$. Motivated by (4), we define an auxiliary operator $H$ in $L_{C}^{2}\left(\Omega, r^{4} \sin \theta / \Delta\right)$ whose domain $D(H)$ coincides with the domain of $A_{0}$ and that is defined by

$$
H f:=\frac{1}{r^{4} / \Delta}\left(-\frac{\partial}{\partial r} \Delta \frac{\partial}{\partial r}-\frac{m^{2} a^{2}}{\Delta}-\frac{1}{\sin \theta} \frac{\partial}{\partial \theta} \sin \theta \frac{\partial}{\partial \theta}+\frac{m^{2}}{\sin ^{2} \theta}\right) f
$$

for every $f \in D(H)$. Utilizing $H$, the identity (4) is equivalent to

$$
\left(A_{0}-\lambda\right) f=T_{r^{4} /(\Delta \bar{\Sigma})}\left(H-\lambda-\lambda T_{\left(\Delta \bar{\Sigma} / r^{4}\right)-1}\right) f
$$

for every $f \in D(H)$. Note that, differently to $A_{0}, H$ can be obtained by "separation" from an operator which is in a certain sense "spherically symmetric." This fact significantly simplifies the study of the properties of $H$.

In the next step, we show that $H$ is a densely defined, linear, symmetric, and essentially self-adjoint operator in $L_{C}^{2}\left(\Omega, r^{4} \sin \theta / \Delta\right)$ which is semibounded from below with lower bound

$$
\beta:=-\frac{m^{2} a^{2}}{r_{+}^{4}} .
$$

In this, $\langle\mid\rangle$ denotes the scalar product of $L_{C}^{2}\left(\Omega, r^{4} \sin \theta / \Delta\right)$. Indeed, it follows for $f, g \in D(H)$, that

$$
\frac{r^{4}}{\Delta} \sin \theta f^{*} H g=\frac{\partial}{\partial r}\left\{\Delta \sin \theta\left[\left(\frac{\partial f}{\partial r}\right)^{*} g-f^{*} \frac{\partial g}{\partial r}\right]\right\}+\frac{\partial}{\partial \theta}\left\{\sin \theta\left[\left(\frac{\partial f}{\partial \theta}\right)^{*} g-f^{*} \frac{\partial g}{\partial \theta}\right]\right\}+\frac{r^{4}}{\Delta} \sin \theta(H f)^{*} g
$$

and hence by Green's theorem that

$$
\langle f \mid H g\rangle=\int_{\Omega} \frac{r^{4}}{\Delta} \sin \theta f^{*} H g d r d \theta=\int_{\Omega} \frac{r^{4}}{\Delta} \sin \theta(H f)^{*} g d r d \theta=\langle H f \mid g\rangle .
$$

Hence, $H$ is symmetric. Further, it follows for $f \in D(H)$ that 


$$
\begin{aligned}
\frac{r^{4}}{\Delta} \sin \theta f^{*}(H-\beta) f= & -\frac{\partial}{\partial r}\left(\Delta \sin \theta f^{*} \frac{\partial f}{\partial r}\right)-\frac{\partial}{\partial \theta}\left(\sin \theta f^{*} \frac{\partial f}{\partial \theta}\right)+\sin \theta\left\{\Delta\left|\frac{\partial f}{\partial r}\right|^{2}+\left|\frac{\partial f}{\partial \theta}\right|^{2}+\left(-\beta \frac{r^{4}}{\Delta}\right.\right. \\
& \left.\left.-\frac{m^{2} a^{2}}{\Delta}+\frac{m^{2}}{\sin ^{2} \theta}\right)|f|^{2}\right\} \geq-\frac{\partial}{\partial r}\left(\Delta \sin \theta f^{*} \frac{\partial f}{\partial r}\right)-\frac{\partial}{\partial \theta}\left(\sin \theta f^{*} \frac{\partial f}{\partial \theta}\right) .
\end{aligned}
$$

Hence it follows by Green's theorem that

$$
\langle f \mid(H-\beta) f\rangle=\int_{\Omega} \frac{r^{4}}{\Delta} \sin \theta f^{*}(H-\beta) f d r d \theta \geq 0
$$

and, finally, that $H$ is semibounded from below with lower bound $\beta$. Hence it follows that $H$ is essentially self-adjoint if there is $\lambda<\beta$ such that the range of $H-\lambda$ is dense in $L_{C}^{2}\left(\Omega, r^{4} \sin \theta / \Delta\right)$. This fact will be proved by application of the theory of Sturm-Liouville operators. For this, we define $I_{r}:=\left(r_{+}, \infty\right)$,

$$
X_{r}:=L_{\complement}^{2}\left(I_{r}, r^{4} / \Delta\right),
$$

and for every $l \in\{|m|,|m|+1, \ldots\}$ the Sturm-Liouville operators $A_{r m l}, A_{r m l 0}$ in $X_{r}$ by

$$
\begin{gathered}
A_{r m l 0} f:=-\frac{1}{r^{4} / \Delta}\left(\Delta f^{\prime}\right)^{\prime}, \\
A_{r m l} f:=\frac{1}{r^{4} / \Delta}\left\{-\left(\Delta f^{\prime}\right)^{\prime}+\left[-\frac{m^{2} a^{2}}{\Delta}+l(l+1)\right] f\right\}=-\frac{1}{r^{4} / \Delta}\left(\Delta f^{\prime}\right)^{\prime}+\frac{1}{r^{4}}\left[l(l+1) \Delta-m^{2} a^{2}\right] f
\end{gathered}
$$

for every $f \in C_{0}^{2}\left(I_{r}, \mathrm{C}\right)$. Obviously, $A_{r m l 0}, A_{r m l}$ are both densely defined, linear, and symmetric. The equation $\left(\Delta f^{\prime}\right)^{\prime}=0$ has nonvanishing constants as solutions. Since these are not in $X_{r}$ at both ends of $I_{r}$, it follows that $A_{r m l 0}$ is in the limit point case at $r_{+}$and at $+\infty$. Hence $A_{r m l 0}$ is essentially self-adjoint (see, e.g., Ref. 18). Further, since $\left[l(l+1) \Delta-m^{2} a^{2}\right] / r^{4}$ is bounded and real-valued, it follows from that by the Rellich-Kato theorem, e.g., see Theorem X.12 in Vol. II of Ref. 15 that $A_{r m l}$ is also essentially self-adjoint, and that the domains of the closures of $A_{r m l 0}$ and $A_{r m l}, \bar{A}_{r m l 0}$, and $\bar{A}_{r m l}$, respectively, coincide. Obviously, $\bar{A}_{r m l 0}$ is semibounded from below with lower bound $\beta$. Hence it follows that the range,

$$
\operatorname{Ran}\left(A_{r m l}-\lambda\right),
$$

of $A_{r m l}-\lambda$ is dense in $X_{r}$ for $\lambda<\beta$. In the following, we assume that $\lambda<\beta$. We note that for every $f \in C_{0}^{2}\left(I_{r}, \mathrm{C}\right)$ and $l \in\{|m|,|m|+1, \ldots\}$,

$$
(H-\lambda)\left[f \otimes\left(P_{l}^{m} \circ \cos \right)\right]=\left[\left(A_{r m l}-\lambda\right) f\right] \otimes\left(P_{l}^{m} \circ \cos \right) .
$$

Also, we denote by $D$ the span of the elements of $D(H)$ of the form

$$
f \otimes\left(P_{l}^{m} \circ \cos \right),
$$

where $f \in C_{0}^{2}\left(I_{r}, \mathrm{C}\right)$ and $l \in\{|m|,|m|+1, \ldots\}$. That $(H-\lambda) D$, and hence also $\operatorname{Ran}(H-\lambda)$, is dense in $L_{C}^{2}\left(\Omega, r^{4} \sin \theta / \Delta\right)$ can be concluded as follows. For this, let $e_{0}, e_{1}, \ldots$ be some Hilbert basis of $X_{r}$. Since $P_{|m|}^{m}{ }^{\circ} \cos , P_{|m|+1}^{m}{ }^{\circ} \cos , \ldots$ is a Hilbert basis of $L_{\mathbb{C}}^{2}\left(I_{\theta}\right.$, sin), where $I_{\theta}:=(0, \pi)$, the family

$$
\left(e_{k} \otimes f_{l}\right)_{(k, l) \in \mathbb{N} \times\{|m|,|m|+1, \ldots\}}
$$

is a Hilbert basis of $L_{C}^{2}\left(\Omega, r^{4} \sin \theta / \Delta\right)$, where

$$
f_{l}:=P_{l}^{m} \circ \cos
$$

for every $l \in\{|m|,|m|+1, \ldots\}$. Since $\operatorname{Ran}\left(A_{r m l}-\lambda\right)$ is dense in $X_{r}$, it follows by (6) that 


$$
e_{k} \otimes f_{l} \in \overline{(H-\lambda) D}
$$

for every $(k, l) \in \mathbb{N} \times\{|m|,|m|+1, \ldots\}$. Since the span of the last family is dense in $L_{\mathrm{C}}^{2}\left(\Omega, r^{4} \sin \theta / \Delta\right)$, this implies that $\overline{(H-\lambda) D}$ is dense in $L_{\mathrm{C}}^{2}\left(\Omega, r^{4} \sin \theta / \Delta\right)$. Hence $H$ is also essentially self-adjoint.

In the final step, we use (5) to prove that

$$
\operatorname{Ran}\left(A_{0}-\lambda\right)
$$

is dense in $X$. Since $H-\lambda$ is essentially self-adjoint such that $\bar{H}-\lambda$ is bijective and since

$$
-\lambda T_{\left(\Delta \bar{\Sigma} / r^{4}\right)-1}
$$

is a positive bounded self-adjoint operator, it follows by the Rellich-Kato theorem that the densely defined, linear, and symmetric operator

$$
H-\lambda-\lambda T_{\left(\Delta \bar{\Sigma} / r^{4}\right)-1}
$$

is essentially self-adjoint and that the closure of this operator is bijective. Hence the range of this operator is dense in $L_{\mathrm{C}}^{2}\left(\Omega, r^{4} \sin \theta / \Delta\right)$ as well as in $X$. Finally, since $T_{r^{4} /(\Delta \bar{\Sigma})}$ defines a bijective bounded linear operator in $X$, it follows by help of (5) that

$$
\operatorname{Ran}\left(A_{0}-\lambda\right)
$$

is dense in $X$. Since $\lambda<\beta \leq \alpha$, the last implies that $A_{0}$ is essentially self-adjoint.

\section{THE CASE OF THE KLEIN-GORDON EQUATION} by

In the case of a Klein-Gordon field of mass $\mu \geq 0$, the equation corresponding to (1) is given

$$
\frac{\partial^{2} u}{\partial t^{2}}+\frac{1}{\bar{\Sigma}}\left(i \frac{4 m M a r}{\Delta} \frac{\partial u}{\partial t}-\frac{\partial}{\partial r} \Delta \frac{\partial}{\partial r}-\frac{m^{2} a^{2}}{\Delta}-\frac{1}{\sin \theta} \frac{\partial}{\partial \theta} \sin \theta \frac{\partial}{\partial \theta}+\frac{m^{2}}{\sin ^{2} \theta}+\mu^{2} \Sigma\right) u=0 .
$$

Hence in this case, the operator corresponding to $A_{0}$ is defined by

$$
A_{0 \mu}:=A_{0}+T_{h},
$$

where $T_{h}$ denotes the maximal multiplication operator in $X$ by the real-valued function $h$ defined by

$$
h:=\mu^{2} \frac{\Sigma}{\bar{\Sigma}}
$$

Since the last is also bounded, $T_{h}$ is a bounded self-adjoint operator on $X$. Hence it follows by the Rellich-Kato theorem that $A_{0 \mu}$ is essentially self-adjoint if and only if $A_{0}$ is essentially selfadjoint. Since the last is the case, $A_{0 \mu}$ is essentially self-adjoint, too.

\section{CONSEQUENCES}

As a consequence of the essential self-adjointness of $A_{0 \mu}$, the objects $X, A_{\mu,-\alpha+\varepsilon}:=A_{\mu}-\alpha+\varepsilon$, $B$, and $C:-(-\alpha+\varepsilon)$ are easily seen to satisfy Assumptions 1 and 4 of Ref. 3. ${ }^{6}$ Here $A_{\mu}$ denotes the closure of $A_{\mu 0}$ and $\varepsilon>0$ is assumed to have the dimension $l^{-2}$. The exact value of $\varepsilon$ does not influence the results in any essential way. In addition, $B$ denotes the maximal multiplication operator in $X$ by the function multiplying $i \partial u / \partial t$ in (7). Since that function is bounded and positive real-valued, $B$ is a bounded linear and positive self-adjoint operator on $X$ given by

${ }^{6}$ See also Sec. 5.1 on "Damped wave equations" in Ref. 5. 


$$
B f=\frac{4 m M a r}{\Delta \bar{\Sigma}} f
$$

for every $f \in X$. Hence, application of the results of Ref. 3 give, in particular, the following well-posed formulation of the initial value problem for (7). ${ }^{8,14}$

\section{Theorem 5:}

(i) By

$$
Y:=D\left(A_{\mu,-\alpha+\varepsilon}^{1 / 2}\right) \times X
$$

and

$$
(\xi \mid \eta):=\left\langle A_{\mu,-\alpha+\varepsilon}^{1 / 2} \xi_{1} \mid A_{\mu,-\alpha+\varepsilon}^{1 / 2} \eta_{1}\right\rangle+\left\langle\xi_{2} \mid \eta_{2}\right\rangle
$$

for all $\xi=\left(\xi_{1}, \xi_{2}\right), \eta=\left(\eta_{1}, \eta_{2}\right) \in Y$, there is a defined complex Hilbert space $(Y,(\mid))$.

(ii) The operators $G$ and $-G$ defined by

$$
G(\xi, \eta):=\left(-\eta, A_{\mu} \xi+i B \eta\right)
$$

for all $\xi \in D\left(A_{\mu}\right)$ and $\eta \in D\left(A_{\mu,-\alpha+\varepsilon}^{1 / 2}\right)$ are infinitesimal generators of strongly continuous semigroups $T_{+}:[0, \infty) \rightarrow L(Y, Y)$ and $T_{-}:[0, \infty) \rightarrow L(Y, Y)$, respectively.

(iii) For every $t_{0} \in \mathbb{R}$ and every $\xi \in D\left(A_{\mu}\right) \times D\left(A_{\mu,-\alpha+\varepsilon}^{1 / 2}\right)$, there is a uniquely determined differentiable map $u: \mathbb{R} \rightarrow Y$ such that

$$
u\left(t_{0}\right)=\xi
$$

and

$$
u^{\prime}(t)=-G u(t)
$$

for all $t \in \mathbb{R}$. Here "' " denotes differentiation of functions assuming values in $Y$. Moreover, this $u$ is given by

$$
u(t):= \begin{cases}T_{+}(t) \xi & \text { for } t \geq 0 \\ T_{-}(-t) \xi & \text { for } t<0\end{cases}
$$

for all $t \in \mathbb{R}$.

\section{ACKNOWLEDGMENTS}

The author is thankful toward an unknown referee for bringing attention to Huzihiro Araki's paper. ${ }^{1}$ In addition, the author is thankful for the hospitality and support by the Sections "Geometric Analysis and Gravitation" (G. Huisken) and "Astrophysical Relativity" (B. F. Schutz) of the Max-Planck-Institute for Gravitational Physics (AEI) and the Institute for Theoretical Astrophysics (TAT, K. Kokkotas) at the University of Tuebingen. This work was supported by the German 
Foundation for Research (DFG) via Grant No. SFB/TR7.

${ }^{1}$ Araki, H., "Selfadjointness and positivity of a certain partial differential operator associated with the Kerr metric in general relativity," Lett. Math. Phys. 18, 355 (1989).

${ }^{2}$ Baker, J., Campanelli, M., Lousto, C. O., and Takahashi, R., "Modeling gravitational radiation from coalescing binary black holes," Phys. Rev. D 65, 124012 (2002).

${ }^{3}$ Beyer, H. R., "A framework for perturbations and stability of differentially rotating stars," Proc. R. Soc. London, Ser. A 458, 359 (2002).

${ }^{4}$ Beyer, H. R., "On the stability of the Kerr metric," Commun. Math. Phys. 221, 659 (2001).

${ }^{5}$ Beyer, H. R., Beyond Partial Differential Equations: A Course on Linear and Quasi-Linear Abstract Hyperbolic Evolution Equations, Springer Lecture Notes in Mathematics 1898 (Springer, Berlin, 2007).

${ }^{6}$ Beyer, H. R. and Craciun, I., "On a new symmetry of the solutions of the wave equation in the background of a Kerr black hole," Class. Quantum Grav. 25, 135014 (2008).

${ }^{7}$ Boyer, R. H. and Lindquist, R. W., "Maximal analytic extension of the Kerr metric," J. Math. Phys. 8, 265 (1967).

${ }^{8}$ Engel, K.-J. and Nagel, R., One-Parameter Semigroups for Linear Evolution Equations (Springer, New York, 2000).

${ }^{9}$ Finster, F., Kamran, N., Smoller, J., and Yau, S.-T., "Decay of solutions of the wave equation in the Kerr geometry," Commun. Math. Phys. 264, 465 (2006).

${ }^{10}$ Kato, T., in Springer Lecture Notes in Mathematics 1540, edited by Komatsu, H. (Springer, Berlin, 1993).

${ }^{11}$ Kay, B. S., "The double-wedge algebra for quantum fields on Schwarzschild and Minkowski spacetimes," Commun. Math. Phys. 100, 57 (1985).

${ }^{12}$ Kay, B. S. and Wald, R. M., "Linear stability of Schwarzschild under perturbations which are non-vanishing on the bifurcation 2-sphere," Class. Quantum Grav. 4, 893 (1987).

${ }^{13}$ Moncrief, V., "Gravitational perturbations of spherically symmetric systems. I. The exterior problem," Ann. Phys. 88, 323 (1974)

${ }^{14}$ Pazy, A., Semigroups of Linear Operators and Applications to Partial Differential Equations (Springer, New York, 1983).

${ }^{15}$ Reed, M. and Simon, B., Methods of Mathematical Physics (Academic, New York, 1975), Vol. I; Methods of Mathematical Physics (Academic, New York, 1980), Vol. II.

${ }^{16}$ Regge, T. and Wheeler, J. A., "Stability of a Schwarzschild Singularity," Phys. Rev. 108, 1063 (1957).

${ }^{17}$ Wald, R. M., "Note on the stability of the Schwarzschild metric," J. Math. Phys. 20, 1056 (1979).

${ }^{18}$ Weidmann, J., Linear Operators in Hilbert Spaces (Springer, New York, 1980).

${ }^{19}$ Zerilli, F. J., "Tensor harmonics in canonical form for gravitational radiation and other applications," J. Math. Phys. 11, 2203 (1970) 